\title{
Responding to Cholera Outbreaks in Zimbabwe: Building Resilience over Time - A Critical Review of Article
}

\author{
N.P. Sithole-Sibanda ${ }^{*}$, L. Nare ${ }^{2}$, D. Chirundu ${ }^{3}$, N.T. Gombe ${ }^{4}$, E. Sibanda ${ }^{1}$ \\ ${ }^{1}$ City of Bulawayo, Health Services Department, Bulawayo, Zimbabwe \\ ${ }^{2}$ Institute of Development Studies, National University of Science and Technology, Bulawayo, \\ Zimbabwe \\ ${ }^{3}$ Kadoma City Health Department, Zimbabwe \\ ${ }^{4}$ African Field Epidemiology Network, Uganda
}

\begin{abstract}
This is a critical review of an article based on the experiences of Epidemic Preparedness and Response pillars as they built resilient health systems while responding to various emerging and reemerging adverse events. The premise of the article is to explore the need and outline advantages of resilient health systems to those responsible for health outcomes at all levels, for effective public health action amidst adverse contexts. The article may be considered as objective and unbiased due to the peer review process. The article was published in a journal that provides a forum for a broad discussion of global health issues that range from systemic violence to infectious disease outbreaks. The article has contributed to literature and provides a basis for achieving resilience, improving public health action and indicates areas for further development of the knowledge base of health systems research. However, it is suggested that the concept of responsiveness be reemphasized and paired with resilience to improve the performance of health systems.
\end{abstract}

Keywords: Emergency, Outbreaks, Response, Resilience, Zimbabwe.

\section{Introduction}

This is a critical review of an article entitled 'Responding to Cholera Outbreaks in Zimbabwe: Building Resilience over Time' published in the 2018 issue of the Current Issues in Global Health Journal. The corresponding author is Chimusoro Anderson. This review will firstly give an overview of the article. Secondly, the article structure will be reviewed on the presentation of the content, the alignment and format of the article. Thirdly, the article will be critiqued looking at the authority, accuracy, currency, relevance, objectivity and coverage. Tables will also be looked at before judging the article's accessibility and credibility.

The central theme of the article is on the importance of building resilient health systems from experiences by Epidemic Preparedness and Response (EPR) pillars as they respond to various disease outbreaks (cholera, Corona Virus Disease of 2019) and disasters. The role of a resilient health system in emergencies response is to ensure that EPR pillars have the capacity to prepare for and effectively respond to crises; maintain core functions when a crisis hits; and, informed by lessons learned during the crisis, reorganise if conditions require it [1]. This occurs amidst economic and persistent system-wide challenges faced by countries in the WHO African region such as Zimbabwe.

\section{Article Summary}

The purpose of the article is to explore the need and outline advantages of resilient health systems. Critical elements that have built resilience include skilled and equipped rapid response teams, strengthened surveillance and maintaining high alert, effective multisectoral collaboration and high-level political engagement [2].

The authors refer to elements of health care systems in Zimbabwe that have become functional through the years, despite the adverse context that includes economic meltdown. The authoritative knowledge that the health workers can provide about the ability to continue operating in challenging environments has contributed to strengthening of the capacity 
of health systems to react to acute external shocks. This could form the basis of collaboration between researchers and health workers, leading to the development and implementation of successful strategies through operational research, to improve health outcomes even within adverse contexts and events such as infectious disease outbreaks.

The article outlines the trend of cholera in Zimbabwe and countries in the WHO African region that were affected by the cholera outbreak. A total of 21 countries in the WHO African region reported cholera outbreaks within the same period of time, reporting a total of 118769 cases and 2531deaths. The overall case fatality rate (CFR) is $2.1 \%$.

The article sets a benchmark for countries with weak health systems, especially lowincome countries, who are seeking to put in place effective measures to control cholera and other disease outbreaks and successfully build resilience to strengthen the capacity of their health systems to respond to outbreaks and crises before, during and after their occur.

\section{Article Structure}

The article was introduced with an abstract which provided the stance or thesis developed by the article as well as a brief overview of main points. The rationales for the article and for the research it describes was also included. The paragraphs in the body were short and therefore the information in each paragraph was easy to access. There were six (6) body headings with detailed information contained under each heading that allows the readers to easily articulate pertinent issues.

The structure of the article is coherent as the article contained the conventional information normally provided in such a study. For example, there are sections related to the background and significance of the research, a review of the literature and methodology used. The dependent and independent variables have been clearly defined. The findings and conclusions were developed towards the end of the article.

The conclusion comprehensively summarized the main points covered by the article and contributed to the development of future policies and research directions regarding resilience of health systems. References were cited in-text and set out clearly in the literature cited section. The article's structure was logically developed overall, with the use of short paragraphs helping the reader access the main points more easily.

The article was HMLT rather than a scanned PDF document and included many links, which helped to make the information accessible. There were links to author, journal, subjects, citations and references which allow the reader to evaluate the articles' worth more effectively. The linked headings and subheadings also allowed the reader to move through the paper more quickly.

\section{Article Critique}

\section{Authority}

The journal, Current Issues in Global Health, is a peer-reviewed scientific journal, which provides a forum for unbiased broad discussions of health issues that range from systemic violence to infectious diseases. The authors come from a variety of environments and strive to promote the maintenance of public health even across international borders, thereby qualifying as issues of global importance. The authors' credibility was established by the fact that the article was peer reviewed. Furthermore, the authors are experienced health practitioners and academics working for the World Health Organization (WHO) in collaboration with the Department of Epidemiology and Disease Control, Ministry of Health and Child Care, Zimbabwe.

\section{Currency}

The article was submitted for publishing on 6 March 2018, reviewed on 27 June 2018 and was accepted for publication on 21 November 2018. The research it describes was current, and the article cites up-to-date references in the body of the text (ranging from 1998 to 2018). Therefore, the article is current.

\section{Relevance}

This was an academic journal on an academic database, which has high credibility in an academic context. The article aimed to encourage countries with weak health systems, especially low-income countries, who are seeking to put in place effective measures to control emerging, re-emerging and rapidly evolving situations such as cholera and the Corona Virus of 2019 (COVID-19) disease 
outbreaks. However, resilience has not been paired with the concept of responsiveness to current and future needs. It is important for resilience (a supply side concept) to be matched by responsiveness if health systems are to effectively serve communities [3].

The article also aimed to commend lowincome countries such as Zimbabwe, that have successfully built resilience through the years as they learnt to positively adapt to adverse contexts and strengthen the capacity of their health systems to respond to outbreaks and crises before, during and after their occur. It helps to inform those responsible for health outcomes at local, provincial, national, regional and international levels on effective public health action rather than to entertain or advertise. It would be relevant to all these groups in their efforts to build resilience as they develop and strengthen the capacity of their health systems to respond to outbreaks or crises and maintain core functions before, during and after an outbreak or crises [4].

\section{Objectivity}

The information was objectively developed, well supported with a current research base and with all evidence acknowledged and referenced. There was no evidence of bias, a fact that was reinforced by the recognition that the article documents research, which followed the rigorous research processes, and the necessary ethical considerations demanded of such community-based research. The authors acknowledged the complexity of issues discussed in a number of ways. For example, the literature review provided explanations of the key terms discussed (for example, 'Resilience') and abbreviations used. The authors supported their research decisions with references from appropriate and relevant literature. The findings are relevant and can be generalized to other countries, especially those with weak health systems and adverse contexts.

\section{Stability}

The article is stable as a resource since it was published in a peer reviewed academic journal on an academic database. It can be depended on for guidance by other researchers and program managers.

\section{Analysis of tables}

There were no graphs in the article. However, one (1) detailed, clear and adequately labeled table was used to compare and describe countries in the WHO African Region that reported cholera outbreaks from January 2017 to March 2018.

\section{Recent advances to the topic}

The concept of 'resilience' is a recent addition to health systems policy and research [5]. It is an achievement in the field of health policy and systems research [4]. A resilient health system is one which is able to effectively prepare for, withstand the stress of, and respond to the public health consequences of disasters [6]. It can also be described as the capacity of health actors, institutions, and populations to prepare for and effectively respond to crisis, maintain core functions when a crisis hits; and, informed by lessons learnt during the crisis, reorganize if conditions require it [1]. Terms that are often used to further describe resilience include adaptation, maintenance, absorption, learning, transformation, withstanding and responding to shocks [7].

Health system resilience underwent a conceptual shift during the Ebola crisis; from a mere 'system' capacity to recognizing the contribution of individuals and their agency within that system and acknowledging the wider social, economic and political context in which responses occur [8]. Resilience and responsiveness are important attributes of an effective health system.

Health systems responsiveness is the ability of the health system to meet the population's legitimate expectations regarding their interaction with the health system, apart from expectations for improvements in health or wealth [9]. Responsive health systems anticipate and adapt to existing and future health needs [10]. Poor responsiveness can also negatively affect utilization of services and the effectiveness of interventions [11].

In recent years, health systems in many lowand middle-income countries have faced severe shocks that range from Ebola epidemics to political and financial crises [4]. Fragile health systems can become overwhelmed during public health crises, further exacerbating the 
human, economic, and political toll [12]. Weak health systems are considered a critical obstacle to improving health [4]. It is therefore important for countries to develop and maintain resilient, responsive health systems that can withstand adverse contexts and events.

Health systems resilience can be achieved, given the competing demands on countries to scale cost-effective health services with scant resources while continuing to be accountable to meet immediate public health needs, both routine and unexpected [13].

Research on resilience of health systems especially in low-income countries is scanty or lacking even as they struggle to function within the context of adverse conditions [2]. Examples of adverse contexts include some of the following: economic meltdown, evidence of instability and daily disruptions, El Niñoinduced drought, lack of access to potable or safe drinking water, climate change, floods and scarce resources [14]. Adverse events include high-consequence infectious diseases such as cholera, Corona Virus Disease of 19 (COVID19), typhoid fever, etc.

Recent global crises have brought into sharp relief the absolute necessity of resilient health systems that can recognize and react to societal crises [15]. This has resulted in resilience

\section{References}

[1]. Kruk, M.E., Myers, M., Varpilah, S.T. \& Dahn, B.T., 2015, What is a Resilient Health System? Lessons from Ebola. The Lancet, 385: 1910-12.

[2]. Chimusoro, A., Maphosa, S., Manangazira, P., Phiri, I., Nhende, T., Danda, S., Tapfumanei, O., Midzi, S.M. \& Nabyonga-Orem, J., 2018. Responding to Cholera Outbreaks in Zimbabwe: Building Resilience over time. Current Issues in Global Health.

[3]. Mills, A., 2017, Resilient and responsive health systems in a changing world, Health Policy and Planning, Volume 32, Issue suppl_3, November 2017, Pages iii1-iii2, https://doi.org/10.1093/heapol/czx117.

[4]. World Health Organization, 2016, Investing in Knowledge for Resilient Health Systems: Strategic Plan 2016-2020.

https://apps.who.int/iris/bitstream/handle/10665/204 806/WHO_HIS_HSR_16.1_eng.pdf?s.

[5]. Witter, S and Hunter, B., 2017, Resilience of health systems during and after crises - what does it becoming the new buzz word in international health policy debates as a way of thinking about how health systems cope with significant external shocks, like the Ebola crisis of 20142015, which is still ongoing. Further research can build on these insights to deepen understanding.

\section{Conclusion}

This was a critical review of Chimusoro et al's article 'Responding to Cholera Outbreaks in Zimbabwe: Building Resilience over Time'. The content, structure, strengths and limitations of the article were analyzed and critiqued. The article has contributed to literature and provides a basis for achieving resilience, improving public health action and indicates areas for further development of the knowledge base of health systems research. It sets a benchmark to other countries seeking to build resilience, develop and strengthen their health systems amidst the context of adversity such as economic meltdown and disease outbreaks such as cholera and the COVID-19 pandemic. However, it is suggested that the concept of responsiveness be re-emphasized and paired with resilience to improve the performance of health systems.

mean and how can it be enhanced? Health systems during and after crisis: evidence for better policy and practice: Brief 1: Rebuild Consortium. https://rebuildconsortium.com/media/1535/rebuild_b riefing_1_june_17_resilience.

[6]. Olu O., 2017, Resilient Health System as Conceptual Framework for Strengthening Public Health Disaster Risk Management: An African Viewpoint. Frontiers in public health, 5, 263. doi:10.3389/fpubh.2017.00263.

[7]. Fridell, M., Edwin, S., von Schreeb, J., \& Saulnier, D. D, 2020, Health System Resilience: What Are We Talking About? A Scoping Review Mapping Characteristics and Keywords. International journal of health policy and management, 9(1), 6-16 https://doi.org/10.15171/ijhpm.2019.71.

[8]. Biddle, L, Wahedi, K, Bozorgmehr, K., 2020, Health system resilience: a literature review of empirical research, Health Policy and Planning, Volume 35, Issue 8, pages 1084-1109, https://doi.org/10.1093/heapol/czaa03. 
[9]. Darby, C, Valentine, N, Murray, C.J.L. \& De Silva, A., 2000, World Health Organization: Strategy on Measuring Responsiveness. GPE Discussion Paper Series No. 23. EIP/GPE/FAR W.H.O.

https://www.researchgate.net/publication/268295796 _World_Health_Organization_WHO_Strategy_on_ Measuring_Responsiveness.

[10]. Mirzoev T, Kane S, 2017, What is health systems responsiveness? Review of existing knowledge and proposed conceptual framework. BMJ Glob Health. 2017;2: e000486. doi:10.1136/ bmjgh-2017-000486.

[11]. Kruk ME, Ling EJ, Bitton A, Cammett M, Cavanaugh K, Chopra M, et al., 2017, Building resilient health systems: a proposal for a resilience index. Biomedical Journal BMJ; 23:357-j2323.

[12]. Nuzzo, J.B., Meyer, D., Snyder, M. et al., 2019, What makes health systems resilient against infectious disease outbreaks and natural hazards? Results from a scoping review. BMC Public Health 19, 1310 (2019). https://doi.org/10.1186/s12889019-7707-z.

[13]. USAID, 2019, From Fragile to Resilient Health Systems: A Journey to Self-Reliance. https://www.usaid.gov/documents/1864/fragileresilient-health-systems-journey-self-reliance.

[14]. Food and Agriculture Organization of the United Nations, 2015, Climate Change and Food Security: Risks and Responses. http://www.fao.org/3/a-i5188e.pdf.

[15]. Gilson L, Barasa E, Nxumalo N, et al., 2017, Everyday resilience in district health systems: emerging insights from the front lines in Kenya and South Africa. BMJ Global Health; 2: e000224. 